cardiometabolic comorbidities associated. Some evidence points out that methotrexate could improve CV risk due to its anti-inflammatory properties, however the effect of PDE4 inhibitor treatment on CV risk have not been elucidated yet.

Objectives: 1) To evaluate the effect of conventional therapy and PDE4 inhibitor in PsA patients with high prevalence of cardiometabolic comorbidities. 2) To identify a molecular patient profile susceptible of being benefit from each therapy regarding disease activity and $\mathrm{CV}$ risk.

Methods: Thirty biological-naïve patients with PsA were treated with the PDE4 inhibitor (Apremilast), Methotrexate or combined therapy (Apremilast and Methotrexate) following the clinical routine practice for 6 months. A cohort of 30 age and sex-matched healthy donors (HDs) was included. Plasma and peripheral blood mononuclear cells (PBMCs) were isolated from peripheral blood of patients and HDs. Different parameters related to the cardiometabolic risk were analyzed, including: atherogenic index, ratio apolipoprotein B (apo B)/apolipoprotein A (apo $A)$, insulin resistance (IR), metabolic syndrome, obesity, arterial hypertension, and the SCORE. Clinical and analytical parameters were collected: lipid profile (total cholesterol, HDL-cholesterol, LDL-cholesterol, triglycerides, apo A and apo $B)$, glucose and insulin, body surface area (BSA) affected by psoriasis, number of tender and swollen joints, DAPSA, C-reactive protein and erythrocyte sedimentation rate. A panel of 92 proteins involved in cardiovascular disease (cardiovascular panel II, Olink) and an adipocytokine profile was measured in plasma and PBMCs. Hard cluster analysis was carried out in order to identify two distinctive molecular phenotypes depending on the treatment response related to the reduction of CV risk.

Results: Among the $92 \mathrm{CV}$-related proteins, the higher levels of two molecules, CD163 and FABP4 observed in PsA patients compared to HDs, were strongly associated with elevated rates of $\mathrm{CV}$ risk factors such as apolipoprotein B/A and atherogenic risks, metabolic syndrome, obesity, IR, arterial hypertension and smoking. Thus, we could identify two clinical profiles of patients according to the plasma levels of these molecules: cluster 1 defined by 20 patients with low levels of CD163 and FABP4 and low prevalence of CV comorbidities and cluster 2 defined by 10 patients with high levels of CD163 and FABP4 and high prevalence of CVD comorbidities. Regarding cluster 2 , those patients that were treated with Apremilast or combined therapy had a significant reduction of CD163 and FABP4 associated with a drop in total cholesterol, apo B, IR state and body mass index. In addition, both PDE4 inhibitor and combined treatment reduced activity disease. However, Methotrexate in monotherapy did not show a beneficial effect in PsA patients displaying higher levels of CD163, FABP4, total cholesterol and no changes in disease activity after treatment. In regard to cluster 1 , the three therapy strategies reduced disease activity after 6 months. Even with low rates of comorbidities, those patients treated with Apremilast had reduced levels of total cholesterol and apolipoprotein B and body mass index after 6 months of therapy. However, no changes were observed in the treatment with Methotrexate or Methotrexate combined with Apremilast.

Conclusion: 1- CD163 and FABP4 could be considered as potential biomarkers of treatment efficacy regarding cardiometabolic comorbidities. 2- Apremilast might target metabolic alterations in PsA modulating lipid profile, insulin resistance and body mass index, decreasing the levels of surrogate CV-related molecules. 3- Apremilast treatment should be considered in PsA patients with higher rates of cardiometabolic comorbidities.

Funded by ISCIII (PI17/01316 and RIER RD16/0012/0015) co-funded with FEDER. Disclosure of Interests: None declared.

DOI: 10.1136/annrheumdis-2021-eular.2956

\section{AB0563 \\ DISCONTINUATION OF ANTI-TNFA IN PATIENTS WITH PSORIATIC ARTHRITIS: A SINGLE-CENTER EXPERIENCE}

D. Graceffa ${ }^{1}$, V. Lora ${ }^{1}$, A. Cristaudo ${ }^{1}$, F. Elia $^{1}$, A. Morrone ${ }^{1}$, C. Bonifati ${ }^{1}$. ${ }^{1}$ San Gallicano Dermatological Institute, IRCCS, Clinical Dermatology, Rome, Italy

Background: TNF inhibitors have been largely demonstrated to be effective and reasonably safe for the treatment of psoriatic arthritis (PSA). Current EULAR guidelines recommend the use of an anti-TNF as first choice treatment in patients with PsA for whom a synthetic DMARD (usually methotrexate or leflunomide) is not efficacious or not well tolerated [1]. In a scenario where biologic treatments are easily available, and the treat to target strategy is widely accepted, a complete disease remission or at least a minimal disease activity are considered realistic goals to be achieved in a growing proportion of patients [2]. However, there remains very little research regarding anti-TNF discontinuation in patients who achieved a complete remission [3-5].

Objectives: The primary aim of this study was to measure the disease-free interval after anti-TNF discontinuation, secondary it was investigated whether the use of Power Doppler Ultrasound (PDUS) and Contrast Enhanced Ultrasound (CEUS) could improve the diagnostic accuracy in the recognition of the relapse. Finally, we wanted to characterize the clinical features of the disease recurrence.

Methods: From June 2018, 35 patients with PsA (27 males and 8 female) treated with anti-TNF, in stable remission were prospectively monitored for 1 year after treatment discontinuation. Remission was defined as documented absence of clinical and ultrasonographic signs of arthritis or enthesitis Complete rheumatological and dermatological examinations were performed in all participants, at baseline and every 8-12 weeks: American College of Rheumatology (ACR) 66-68 joint count; Psoriasis Area Severity Index (PASI); patient pain visual analog score (VAS); patient global disease activity VAS; Health Assessment Questionnaire (HAQ); Leeds Enthesitis Index (LEI); Bath Ankylosing Spondylitis Disease Activity Index (BASDAI); Bath Ankylosing Spondylitis Functional Index (BASFI); Power Doppler Ultrasound (PDUS) of the involved joints and entheses, Contrast Enhanced Ultrasound (CEUS) of a selected joint or enthesis and laboratory inflammation tests.

Results: 31 out of the 35 enrolled patients, experienced a disease recurrence with an average disease-free interval of $27.9 \pm 21.1$ weeks (Figure 1) In 3 patients the treatment was restored for a relapse of the skin psoriasis, 8 patients reported only axial symptoms of disease relapse and 20 patients had both axial and peripheral joints involvement (average DAPSA score of $23.6 \pm 11.1$; average BASDAI score of $4.7 \pm 2.6$; average BASFI score $4.5 \pm 2.9$ ) In all cases the disease flare was moderate and all patients promptly regained remission after restarting the treatment. Both PDUS and CEUS were safe and reliable showing a good percentage of accordance $(95,4 \%)$ in detecting synovitis and enthesitis.

Conclusion: The rate of disease relapse of PsA after anti-TNF discontinuation is relevant. However the disease-free interval was not short. Retreatment with the same anti-TNF was effective and safe.

\section{REFERENCES:}

[1] Gossec L, Baraliakos X, Kerschbaumer A, et al. EULAR recommendations for the management of psoriatic arthritis with pharmacological therapies: 2019 update. Ann Rheum Dis. 2020 Jun;79(6):700-712.

[2] Dures E, Shepperd S, Mukherjee S, et al. Treat-to-target in PsA: methods and necessity. RMD Open. 2020 Feb;6(1):e001083.

[3] Stober C, Ye W, Guruparan T, et al. Prevalence and predictors of tumour necrosis factor inhibitor persistence in psoriatic arthritis. Rheumatology (Oxford). 2018 Jan 1;57(1):158-163.

[4] Huynh DH, Boyd TA, Etzel CJ, et al. Persistence of low disease activity after tumour necrosis factor inhibitor (TNFi) discontinuation in patients with psoriatic arthritis. RMD Open. 2017 Jan 16;3(1):e000395.

[5] Ye W, Tucker LJ, Coates LC. Tapering and Discontinuation of Biologics in Patients with Psoriatic Arthritis with Low Disease Activity. Drugs. 2018 Nov;78(16):1705-1715

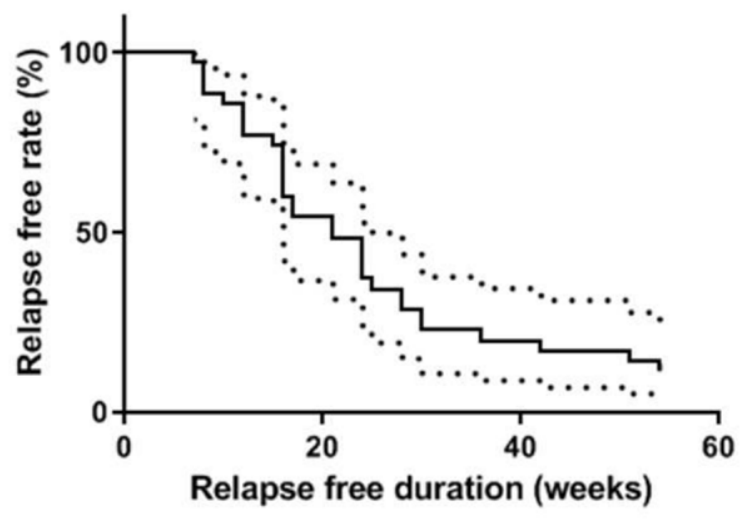

Fig.1 Relapse-free rates after discontinuing anti-TNF. Kaplan-Meier curve demonstrated the disease free interval after biologic cessation.

Disclosure of Interests: None declared.

DOI: 10.1136/annrheumdis-2021-eular.3002

\begin{tabular}{|l|l}
\hline AB0564 & RHEUMATOLOGISTS' VIEWS AND EXPERIENCES IN \\
MANAGING PSA PATIENTS BASED ON TREATING TO \\
TARGET STRATEGY: A CROSS-SECTIONAL STUDY IN \\
CHINA
\end{tabular}

L. Xu ${ }^{1}$, Z. Wang ${ }^{1}$, J. Xue ${ }^{1}$, M. Bai ${ }^{1}$, H. Zhong ${ }^{1}$, Y. Su ${ }^{1} .{ }^{1}$ Peking University People's Hospital, Department of Rheumatology and Immunology, Beijing, China 